\title{
Conservación de objetos metálicos: examen, formas de corrosión y la "superficie original": un curso teórico-práctico (ENCRyM- INAH, México, noviembre 2014)
}

Conservation of Metal Objects: Examination, Forms of Corrosion and "the Original Surface": A Theoretical and Practical Course (ENCRyM-INAH, Mexico, November 2014)

\author{
Luisa Straulino Mainou \\ Centro INAH Quintana Roo, \\ Instituto Nacional de Antropología e Historia (INAH), México \\ azucarylimon@gmail.com
}

\section{Resumen}

El Seminario-Taller en Conservación de Patrimonio Metálico de la Escuela Nacional de Conservación, Restauración y Museografía (ENCRyM), Instituto Nacional de Antropología e Historia (INAH), México, organizó el curso Conservación de objetos metálicos: examen, formas de corrosión y la "superficie original" del 18 al 21 de Noviembre del año 2014, que impartió el doctor Regis Bertholon, profesor de la Haute École de Conservation-Restauration Arc, Suiza. Los tópicos principales fueron mecanismos de corrosión en aleaciones de hierro, cobre y plata, descripción de capas de corrosión y localización del límite de la superficie original. Esta RESEÑA analiza el contexto, contenido y alcances de esta experiencia de actualización profesional en México.

\section{Palabras clave}

conservación; metales; corrosión: limite de la superficie original; Regis Bertholon; curso de actualización

\section{Abstract}

As part of the Seminar-Workshop Conservation of Metallic Heritage, Escuela Nacional de Conservación, Restauración y Museografía (ENCRyM, National School of Conservation, Restoration and Museum Studies), Instituto Nacional de Antropología e Historia (INAH, National Institute of Anthropology and History), Mexico, doctor Regis Bertholon, from the Haute École de ConservationRestauration Arc, Switzerland, gave a course entitled Conservation of Metal Objects: Examination, Forms of Corrosion and "the Original Surface" (18-21 November 2014). Main topics were 
corrosion mechanisms of iron, copper and silver alloys, the description of corrosion layers as well as the localization of the original limit of the surface. This REVIEW critically analyses the context, content and scope of this refresher course experience.

\section{Key words}

conservation; metals; corrosion; limit of the original surface; Regis Bertholon; refresher course

\section{Introducción}

E I curso Conservación de objetos metálicos: examen, formas de corrosión y la "superficie original" (Figura 1) que impartió el doctor Regis Bertholon del 18 al 21 de noviembre de 2014 en la Escuela Nacional de Conservación, Restauración y Museografía del Instituto Nacional de Antropología e Historia (ENCRyM-INAH) fue organizado por las profesoras encargadas del Seminario-Taller de Conservación de Patrimonio Metálico de dicha escuela: las restauradoras Janeen Contreras Vargas, Gabriela Peñuelas Guerrero y Marcela López Arriaga, quienes cuentan con una amplia experiencia docente nacional e internacional en la materia (Peñuelas Guerrero et al. 2011).

Gracias a que se difundió que se iba a impartir dicho curso en varios medios, entre ellos las redes sociales, postulé mi candidatura para ser aceptada. Mi motivación procedió tanto del ámbito de mi interés profesional como de las necesidades institucionales del Centro INAHQuintana Roo, donde laboro y en el que resulta pertinente la actualización constante de conocimientos y habilidades en lo que se refiere a la conservación de patrimonio metálico, un tipo de bien cultural que, a la par de la cerámica, piedra y estuco, conforma importantes colecciones a resguardo del centro. Hoy en día las posibilidades de capacitación profesional en conservación-restauración son escasas en México, y aún más las revisiones que encontramos en la literatura. Por lo tanto, dedico los siguientes párrafos a reseñar el curso, perfilando primero la trayectoria de Bertholon, para posteriormente detenerme en algunas particularidades de la estructura: contenido, forma de la experiencia de enseñanza-aprendizaje y finalizar con algunas reflexiones sobre su pertinencia, limitaciones y perspectivas.

\section{Desarrollo}

Regis Bertholon es un reconocido experto de la conservación del patrimonio metálico. Además de su trayectoria profesional como investigador, destaca su papel como profesor de la Universidad París-1 (Pantheon Sorbonne, Francia), fundador del departamento de conservación de metales en el Institut National du Patrimoine (Francia) y actual director

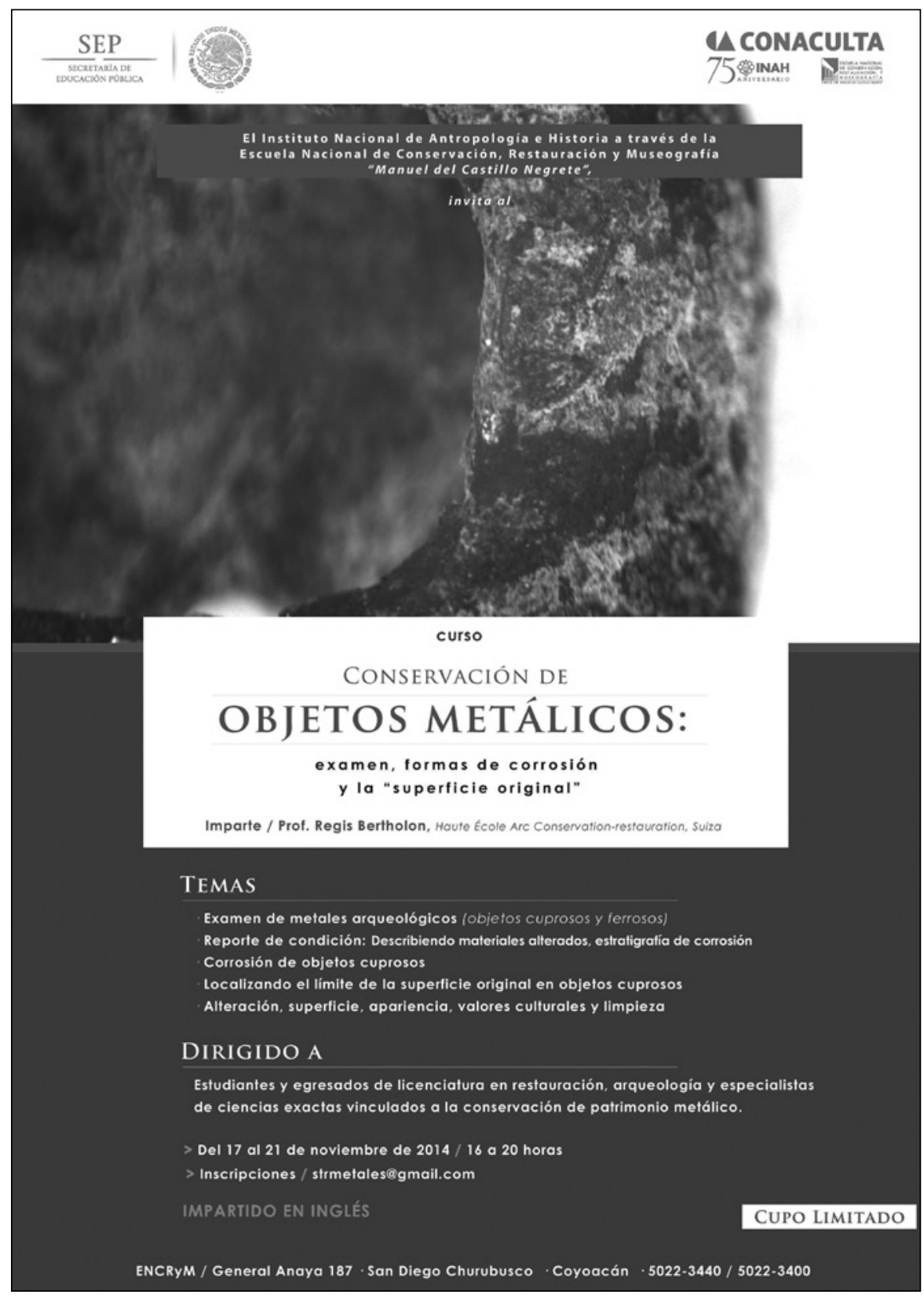

FIGURA 1. Cartel del curso Conservación de objetos metálicos: examen, formas de corrosión y la "superficie original" , ENCRyM-INAH, México (Diseño: Anacani Ramón, 2014; cortesía: ENCRyMINAH). 
del Departamento de Conservación y Restauración en la Haute École de Conservation-restauration Arc (HEArc) en Neuchâtel, Suiza (SNIS 2014). Su amplio repertorio hemerobibliográfico abarca revistas y libros científicos consagrados a sus investigaciones sobre métodos para describir la corrosión en metales arqueológicos y localizar el límite de la superficie original de los metales después de su alteración (de lo que hablaré con detalle más adelante); metodologías de conservación; disertaciones sobre los valores culturales y aportaciones a la historia de la conservación y restauración (Bertholon 1993, 2000, 2001a, 2001b, 2001c, 2001d, 2001e, 2002a, 2002b, 2004; BerthoIon y Pain 1987, 1988; Bertholon y Relier 1987, 1988; Bertholon et al. 1995, 1998).

Profesionalmente, Bertholon se inscribe en un marco global de conservadores de patrimonio metálico, y en la escena francesa se consideraría sucesor de Albert France-Lanord (1915-1993), quien, junto con otros investigadores, desarrolló las técnicas de restauración, conservación y estudio de los objetos metálicos arqueológicos en el Laboratoire d'Archéologie des Mataux (Nancy, Francia), y además se ocupaba de temas de ética en la conservación (France-Lanord 1980, 1996). Otras referencias importantes son, sin duda, los textos de Scott et al. (1994), Meyer-Roudet (1999) y Dillman et al. (2013), que se dedican a recopilar trabajos sobre análisis en laboratorio del patrimonio metálico, con tecnología aplicada a la conservación de patrimonio cultural, y los procesos de conservación aplicados en Europa y Estados Unidos. En un contexto más cercano a México (Peñuelas-Guerrero et al. 2013; Contreras et al. 2013) y España (Barrio et al. 2007; Blasco et al. 2007) se han conformado grupos interdisciplinarios aplicados al estudio y conservación de patrimonio metálico, los cuales han generado, entre otros productos académicos, un diplomado de especialización organizado por parte de la Universidad Autónoma de Madrid (UAM, España) y la ENCRyM-INAH (Tapia 2013). En este universo de especialidad destaca el trabajo de Berdescripción, el análisis y la conservación de los metales arqueológicos sino que a partir de ellos desarrolla una propuesta teórico-metodológica con aplicación práctica.

La oportunidad de contar en México con un profesor que se distingue por ser una persona sencilla $y$ de buen humor, que ama la tarea de enseñar y discutir el quehacer de la conservación y la restauración, era única (Figura 2). Una de las aportaciones más significativas del curso fue la reiteración de que "el conocimiento se construye en conjunto".

Éste se distinguió por las diversas modalidades de enseñanza-aprendizaje: por un lado, clases formales, trabajo en laboratorio y seminarios de trabajo con una temática igualtholon porque no sólo se ocupa de la

mente amplia, que compendió el análisis visual y la descripción de alteraciones en artefactos metálicos; los mecanismos de corrosión de cobre, hierro y plata; la localización del límite de la superficie original y, por el otro, discusiones abiertas sobre la apariencia superficial, su relación con la limpieza y la consideración de los valores de los objetos en virtud de la toma de decisiones en conservación. De lo mencionado subrayo y desarrollo dos temas que, al ser dos aportaciones originales de Bertholon, constituyeron el corazón de su curso: el método de descripción de la corrosión y el concepto de límite de la superficie original.

Para Bertholon la descripción adecuada (metódica y estandarizada) de los objetos metálicos y sus capas de corrosión es esencial porque favorece la transmisión de conocimiento entre profesionales no sólo de la conservación y la restauración,

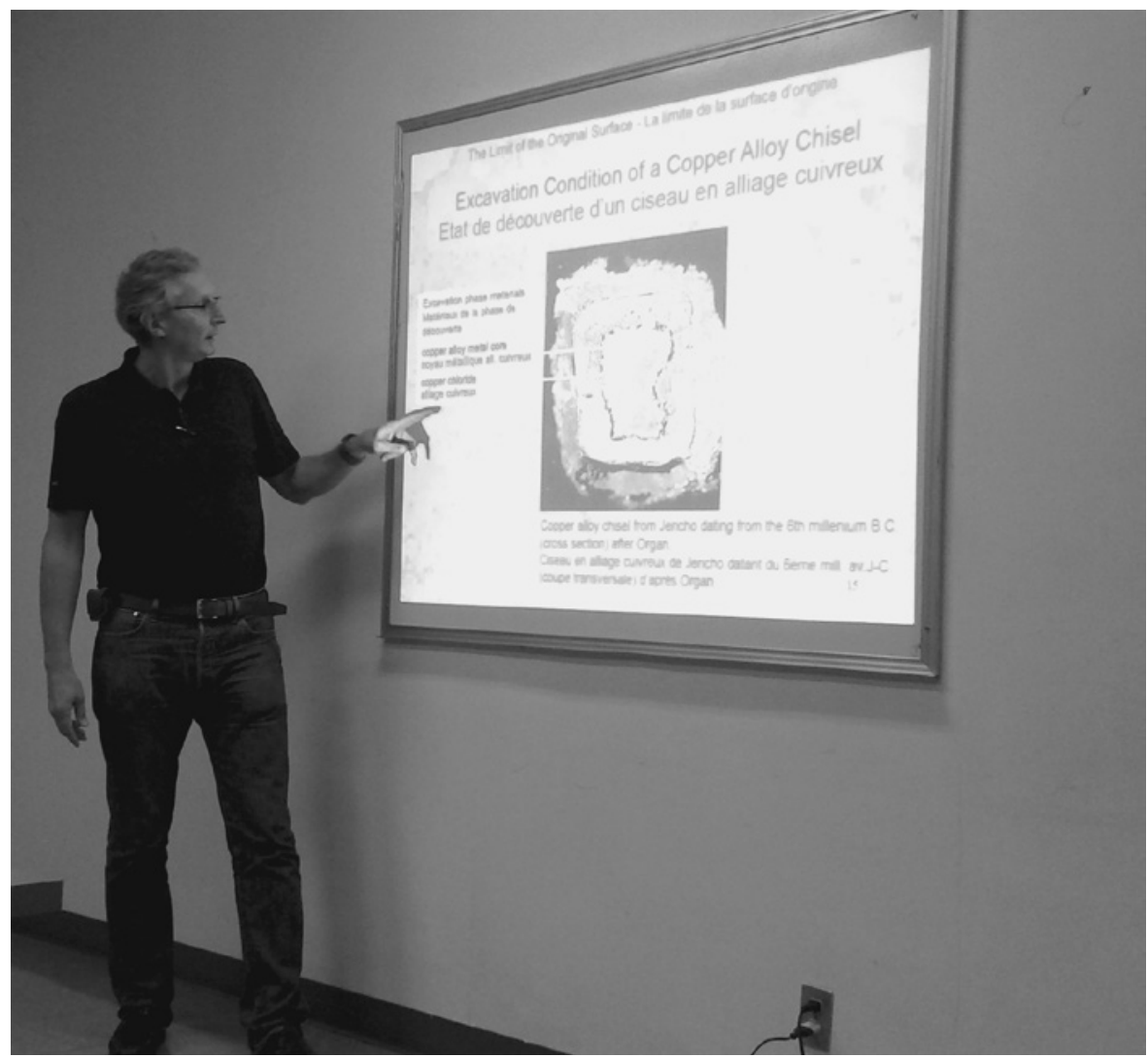

FIGURA 2. El doctor Regis Bertholon imparte el curso Conservación de objetos metálicos: examen, formas de corrosión y la "superficie original", ENCRyM-INAH, México (Fotografía: Janeen Contreras; cortesía: Seminario-Taller de Conservación de Patrimonio Metálico, ENCRyM-INAH). 
sino de otras disciplinas como arqueometría, arqueología, etc. Así, no sólo los productos de corrosión son el objeto de interés, también se le da importancia a las formas de corrosión, de modo que la descripción se vuelve un puente importante entre las observaciones macroscópicas y las realizadas con microscopios estereoscópicos y ópticos, y los estudios más detallados como observación e identificación con microscopia electrónica de barrido con microsonda acoplada, PIXE, FTIR, etcétera.

La base principal del método de descripción que propone Bertholon para estudiar las formas de corrosión es la división virtual de diferentes volúmenes, Ilamados estratos, cada uno de los cuales tiene interfases, entendidas de dos formas: como límites en el sentido matemático, y como una sección diferente del material adyacente, con estructura y composición distinta de las capas inmediatamente inferiores y superiores. La descripción comienza desde la capa superficial hacia el núcleo y comprende varios aspectos: su posición (paralelas o perpendiculares a la superficie), definición de las características del estrato (morfología, apariencia, microestructura, textura, composición) y estructura (relación entre los estratos e interfases) (BerthoIon 2000:225-295). Las descripciones detalladas de estratos e interfases y la comprensión de los mecanismos de corrosión conducen a la obtención de pistas valiosas para localizar el límite de la superficie original (Iimit of the original surface o limitos). Pero ¿qué es el limitos?

De inicio, se trata de un concepto -que figura entre las contribuciones claves de Bertholon- cuya comprensión exige desarrollar las ideas detrás del concepto: a) la superficie original de un artefacto se define como la superficie de éste al tiempo de ser abandonado; el abandono, deliberado o no, da fin al periodo de modificaciones antrópicas antes de su excavación; $b$ ) debido a varios factores, la superficie original de un artefacto cambia desde que deja de utilizarse, por lo que la actual, sin importar sus procesos ni sus grados de corrosión, no es ya más la superficie original; c) como no es posible recuperar la superficie original, dado que ha sido alterada o destruida, la alternativa consiste en intentar localizar el limitos, que, como límite (valga la redundancia), es abstracto: en algunos casos corresponde a la interfase entre productos de corrosión; en otros a la superficie presente, y en algunos más no es posible distinguirlo con certeza (Bertholon 2004:171).

Puesto que la identificación del limitos es materia de análisis y discusión, parte del curso se dedicó a analizar la corrosión de bienes culturales metálicos con el fin de reflexionar e intentar identificar el limitos en función de indicadores. Casi siempre es posible distinguir marcadores del límite de la superficie original que se dividen en: a) marcadores superiores del limitos que son algunas características de una capa de corrosión que indica que el limitos está bajo ella, b) y los marcadores inferiores que indican que el limitos está sobre ella; c) un tercer tipo lo constituye aquel en que las características de una interfase indican que ésta corresponde al limitos (tablas de estos marcadores se pueden encontrar en Bertholon 2004:175-179). No obstante, dada la relativa validez de estos marcadores, ha de confrontarse y discutirse la pertinencia de su identificación en función del metal y sus condiciones de corrosión. Además de que la bibliografía ofrece ejemplos donde se pone en práctica la localización del limitos (Bertholon 2001b), una aportación fundamental del curso fue la posibilidad de discutir entre los asistentes - con diversas trayectorias- observaciones, marcadores y conclusiones sobre el limitos en el marco de la gran experiencia y conocimiento que posee Bertholon.

Aunado a lo anterior, valga resaltar las ventajas de la pedagogía empleada en el curso, fundada en un proceso de aprendizaje inductivo, que comenzó por describir corrosio- nes en piezas específicas para después tomar la clase de descripción metodológica y volver sobre nuestros pasos.

En otro aspecto, anoto algunas limitaciones del curso: por un lado, la discusión sobre alteraciones, apariencia superficial, limpieza y valores culturales fue muy breve, sobre todo si se considera la dificultad de expresar/comprender en inglés ideas complejas asociadas con la teoría, la ética y la valoración en materia de conservación; por el otro, hubiera sido interesante trabajar con objetos de cobre sacrificables (en lugar de objetos de cobre prehispánicos), como lo hicimos con los de hierro, para desarrollar de manera más sencilla el trabajo de identificación y descripción de las capas de corrosión.

\section{Reflexiones finales}

En suma, un curso más largo y con más posibilidades prácticas ofrecería grandes ventajas, pues sería importante trabajar no sólo con observaciones macroscópicas y microscopios estereoscópicos en muestras sin preparar, sino también combinando diferentes técnicas de análisis micromorfológicos, elementales y de identificación de compuestos para la descripción, y en particular desarrollar preparaciones metalográficas para el estudio y descripción de las capas de corrosión. Asimismo sería provechosa una exposición más extensa de casos particulares de intervenciones no convencionales, y el ejercicio de generar propuestas para un caso particular que se discutieran en el grupo de trabajo.

Enfatizo que el curso hubiera resultado de interés no sólo para quienes son restauradores de patrimonio metálico, sino para cualquier interesado en la arqueometría de los metales (arqueólogos, químicos, fisicos, etc.), ya que proporcionó una metodología clara, ordenada y estandarizada para estudiar la corrosión como un sistema formado por capas que tiene el potencial de ser adaptado por restauradores y profesionales 
afines para analizar otros materiales estratificados. De ahí que sea deseable y necesario el pronto regreso de este especialista para la actualización de profesionales del estudio y la conservación de patrimonio cultural en general, y el metálico en particular. Ojala que así suceda.

\section{Referencias}

Barrio Martín, Joaquín, Jorge Chamón, Marco Ferretti, Margarita Arroyo, Ana Isabel Pardo et al.

2007 "Study of the conservation problems of the archaeological gilded metals from the Islamic site of Qalat Rabah", Proceedings of the International Congress Conservation of Metal ICOM-CC, Ámsterdam, Rijmuseum, 1727 de septiembre, preprints, I: 10-16.

Bertholon, Regis

1993 "Corrosion du cuivre et des ses alliages lors d'un traitement de désinfestation par fumigation à partir de phosphine", Actes de la 7e Rencontre Annuelle du Groupe de Travail, Draguignan, París, Groupe ICOM-CC-SFIIC, Section métal: 1-13.

2000 "La limite de la surface d'origine des objets métalliques archéologiques. Caractérisation, localisation et approche des mécanismes de conservation", tesis de doctorado en humanidades y ciencias sociales, Université Panthéon-Sorbonne-París 1.

2001a "Nettoyage et stabilisation de la corrosion par électrolyse: le cas des canons provenant de fouilles sousmarine", en Claude Volfovsky (ed.), La conservation des métaux, París, CNRS (Conservation du patrimoine, 5), 83101.

2001b "Characterization and location of the original surface of corroded archaeological objects", Surface Engineering, 17 (3): 241-245.

2001c "Nettoyage et consolidation des armes en fer du sanctuaire celtique de Gournay-sur-Aronde", en Claude Volfovsky (ed.), La conservation des métaux, París, cNRS (Conservation du patrimoine, 5), 103-112. 2001d "Nettoyage d'une plaque-boucle mérovingienne", en Claude Volfovsky (ed.), La conservation des mé- taux, París, CNRS (Conservation du patrimoine, 5), 113-118.

2001e "Les bronzes Omeyyades de Mafraq (Jordanie): stabilisation de la corrosion", en Claude Volfovsky (ed.), La conservation des métaux, París, CNRS (Conservation du patrimoine, 5), 119-133.

2002a "Proposition d'une méthode de description de la corrosion adaptée aux objets métalliques archéologiques: schéma général de la méthode", La conservation-restauration en archéologie. Urgences: problématiques et applications. Actualité XVII e journées des restaurateurs en archéologie, (Conservation-restauration des biens culturels. Cahier technique), París, ARAAFU, 9: 56-65.

2002b "Soustractions et conservation: de l'intérêt de la conservation-restauration dans l'étude archéologique des objets", Annales d'histoire de l'art \& d'archéologie, 24: 95-106.

2004 "The location of the original surface: a review of the conservation literature", en I. D. MacLeod, J. M. Theile y C. Degrigny (eds.), Metal 2001: Actas del Congreso Internacional sobre la Conservación de Metales: Santiago, Chile, abril 2001, Freemantke, Western Australia Museum: 167-179.

Bertholon, Regis, L. Garenne-Marot, J. M. Blengino y E. Durand

1995 "De nouvelles approches de la lecture du rouleau de cuivre de Qumran: re-restauration minimale, radiographie $X$ et traitement $d^{\prime}$ image, moulage et galvanoplastie", en Memoirs Colloque sur la Conservation Restauration des Biens Culturels: Restauration, dé-restauration, re-restaurati, París, ARAAFU, 4: 295-306.

Bertholon, Regis y Silvia Pain

1987 "L'électrodialyse appliquée à la déchloruration des métaux ferreux", Journées sur la conservation, restauration des biens culturels: recherches et techniques actuelles, París, 15-16 octobre.

1988 Le traitement électrolytique des objets archéologiques en alliages cuivreux. Premiers resultats, Saint-Denis, Electricité de France.

Bertholon, Regis y C. Relier

1987 "Les métaux ferreux et non fe- rreux", en Conservation-restauration du mobilier archeologique, Saint-Denis, L'unite archeologique de la ville de Saint-Denis, 41-50.

1988 "Le probleme des métaux archeologiques", en N. Meyer y C. Relier, Conservation des sites et du mobilier archeologiques: Principes et methods (Etudes et documents sur le patrimoine culturel), París, UNESCO, 15: 60-69.

Bertholon, Regis, L. Robbiola y N. Lacoudre

1998 "Corrosion du rouleau de cuivre de Qumrân et localisation de la surface originelle", Metal 98: Proceedings of the International Conference on Metals Conservation, DraguignanFiganières, Francia, 27-29 de mayo.

Blasco, Concepción, Joaquín Barrio y Pilar Pineda

2007 "La revitalización de los ritos de enterramiento y la implantación de la necrópolis de incineración en la cuenca del Manzanares: la necrópolis de Arroyo Butarque (Madrid)", Zona Arqueológica, 10 (II): 216-238.

Contreras Vargas, Janeen, José Luis Ruvalcaba-Sil y F. J. Rodríguez-Gómez

2013 "Effects of the cleaning of silver with acidified thiourea solutions", en Metal 2013, Interim Meeting of the ICOM-CC Metal Working Group Conference Proceedings, Edinburgo, Escocia, 16-20 de septiembre.

Dillmann, P., D. Watkinson, U. E. Ange-

lini y A. Adriaens

2013 Conservation of Cultural Heritage Metallic Artefacts, Cambridge, Woodhead.

France-Lanord, Albert

1980 Ancient Metals. Structure and Characteristics. Technical Cards/Métaux Anciens. Structure et Caractéristiques. Fiches Techniques English, Roma, ICCROM.

1996 "How to question an object before restoring it", en Nicholas Stanley Price, Mansfield Kirby Talley y Alessandra Melucco (eds.), Historical and Philosophical Issues in the Conservation of Cultural Heritage, Los Ángeles Getty Conservation Institute, 244-247.

Meyer-Roudet, H. (ed.)

1999 Á la recherche du metal perdu, París, Errance. 
1991 "La déchloruration des alliages cuivreux par électrolyse à faible polarisation dans le sesquicarbonate de sodium", Studies in Conservation, 36 (1): 33-43.

Peñuelas-Guerrero, Gabriela, Janeen Contreras Vargas y María del Pilar Tapia López (comps.)

2011 Notas corrosivas. Memorias del $3^{\text {er }}$ Congreso Latinoamericano de Restauración de Metales, México, ENCRyMINAH.

Peñuelas-Guerrero, Gabriela, Janeen Contreras Vargas, Niklas Schulze, José Luis Ruvalcaba-Sil y Edith Ortiz-Díaz

2013 "Review of ethical considerations in conserving and analysing mexican metallic pre-Columbian artefacts", en Metal 2013, Interim Meeting of the ICOM-CC Metal Working Group Conference Proceedings, Edinburgo, Escocia 16-20 de septiembre.

Scott, D. A., J. Podany y B. B. Considine (eds.) 1994 Ancient \& Historic Metals: Conservation and Scientific Research: Proceedings of a Symposium Organized by the J. Paul Getty Museum and the Getty Conservation Institute, November 1991, Marina del Rey, Getty Conservation Institute, documento electrónico disponible en [http://hdl.handle.net/10020/gci_pubs/ancientmetals], consultado en febrero de 2015.

SNIS (Swiss Network for International Studies)

2014 CV Dr. Regis Bertholon, documento electrónico disponible en [http://www.snis.ch/content/regis-bertholon], consultado en enero de 2015.
Tapia López, María del Pilar

2013 "Entre la arqueología y la restauración. Diálogos con Joaquín Barrio Martín", Intervención, Revista Internacional de Conservación, Restauración y Museología, 7: 5-10.

\section{Síntesis curricular del/os autor/es}

\section{Luisa Straulino Mainou}

Centro INAH Quintana Roo,

Instituto Nacional de Antropología e Historia (INAH), México

azucarylimon@gmail.com

Licenciada en restauración (Escuela Nacional de Conservación, Restauración y Museografía [ENCRyM], Instituto Nacional de Antropología e Historia [INAH], México), galardonada con la mención honorífica del Premio INAH "Paul Coremans" (México, 2009). Ha trabajado en el área de conservación de patrimonio arqueológico en zonas como Templo Mayor, Tlatelolco, Río Bec y a partir de 2010 es restauradora de base del INAH, delegación Quintana Roo, todas en México. Actualmente se encuentra estudiando la maestría en estudios mesoamericanos (Facultad de Filosofía y Letras [FFyL], Universidad Nacional Autónoma de México [UNAM], México).

Intervención, Revista Internacional de Conservación, Restauración y Museología, año 6, número 11, enero-junio de 2015, se terminó de imprimir en junio de 2015, en los talleres Calle México, D.F.

La edición consta de 1000 ejemplares, impresos en papel cultural ahuesado de 90 gr para interiores, y en couché de 250 gr para los forros. 